\title{
Activité Antioxydante Et Teneur En Flavonoïdes De Cinq Plantes De La Famille Des Fabaceae Utilisées Contre De l'Ostéoporose Au Centre De La Côte d'Ivoire
}

\author{
Kouassi Kouadio Aubin, Docteur \\ Kouadio N'guessan Jules, Assistant
}

Laboratoire de Botanique et Valorisation des Ressources Végétales, Sciences de la Nature, Université Nangui Abrogoua, Abidjan, Côte d'Ivoire

\section{Ahoua Angora Rémi Constant, Docteur}

Centre Suisse de Recherches Scientifiques en Côte d'Ivoire, Abidjan

\section{Yao Konan, Attaché de Recherche}

Centre National de Floristique,

Université Félix Houphouët-Boigny, Abidjan, Côte d'Ivoire

Centre Suisse de Recherches Scientifiques en Côte d'Ivoire, Institut Botanique Aké-Assi d'Andokoi, Abidjan, Côte d'Ivoire

\section{Kanga Yao, Assistant}

Université Peleforo Gon Coulibaly de Korhogo, UFR Sciences Biologique

\section{Moya Bi George, Doctorant}

Laboratoire de Botanique et Valorisation des Ressources Végétales, Sciences de la Nature, Université Nangui Abrogoua,Côte d'Ivoire

\section{Koné Mamidou Witabouna, Professeur}

Laboratoire de Botanique et Valorisation des Ressources Végétales, Sciences de la Nature, Université Nangui Abrogoua, Côte d'Ivoire Centre Suisse de Recherches Scientifiques en Côte d'Ivoire, Abidjan

\section{Résumé}

La déficience hormonale due à l'âge entraîne entre autres une augmentation de la fragilité osseuse. Pour lutter efficacement contre cette affection, de nombreux auteurs ont préconisé l'usage des plantes. Dans le but d'apporter une contribution à la freinée de cette pathologie, cette étude a été menée en vue de valoriser des plantes médicinales riches en phytostrogènes comme une solution alternative contre l'ostéoporose. Pour ce faire, les teneurs en flavonoïdes et isoflavones de cinq plantes issues de la famille des Fabaceae ont été déterminées par les méthodes spectrophométriques. Aussi a été évalué le pouvoir anti-radicalaire de ces plantes en utilisant les méthodes au DPPH et 
à l'ABTS. La présente étude indique la présence d'isoflavones et flavonoïdes dans les extraits de plantes à l'exception de celui des racines de Piliostigma thonningii. Les feuilles de Uraria picta possèdent les plus fortes teneurs de flavonoïdes avec 334,48 $\pm 0,49 \mathrm{mg}$ EQ $/ 100 \mathrm{~g}$ d'extrait, suivies de celles de Pericopsis laxiflora avec 210,27 $\pm 0,71 \mathrm{mg}$ EQ /100g d'extrait. Ces deux plantes présentent également des teneurs élevées en isoflavones avec respectivement $66,61 \pm 1,23 \mathrm{mg}$ GNT $/ 100 \mathrm{~g}$ d'extrait et $21,05 \pm 1,15 \mathrm{mg}$ GNT $/ 100 \mathrm{~g}$ d'extrait. Les feuilles, les racines et l'écorce de tige de Pericopsis laxiflora présentent les plus fortes activités antioxydantes avec respectivement, $95,86 \pm 9,27 \%, 91,02 \pm 0,58 \%$ et $99,99 \pm 0,0 \%$ d'inhibition pour le radical DPPH et respectivement $100 \pm 0,00 \%, 92,53 \pm 1,26 \%$ et $99,99 \pm 0,01$ $\%$ d'inhibition pour le radical ABTS. Les résultats montrent que ces plantes peuvent avoir un intérêt dans la prévention et/ou le traitement de l'ostéoporose.

Mots clés : Plantes Médicinales, Flavonoïdes, Isoflavones, Antioxydants, Fabaceae, Ostéoporose 


\title{
Antioxidant Activity and Flavonoid Content of Five Plants of the Fabaceae Family Used Against Osteoporosis in Central Côte d'Ivoire
}

\author{
Kouassi Kouadio Aubin, Docteur \\ Kouadio N'guessan Jules, Assistant
}

Laboratoire de Botanique et Valorisation des Ressources Végétales, Sciences de la Nature, Université Nangui Abrogoua, Abidjan, Côte d'Ivoire

\section{Ahoua Angora Rémi Constant, Docteur}

Centre Suisse de Recherches Scientifiques en Côte d'Ivoire, Abidjan

\section{Yao Konan, Attaché de Recherche}

Centre National de Floristique,

Université Félix Houphouët-Boigny, Abidjan, Côte d'Ivoire

Centre Suisse de Recherches Scientifiques en Côte d'Ivoire, Institut Botanique Aké-Assi d'Andokoi, Abidjan, Côte d'Ivoire

\section{Kanga Yao, Assistant}

Université Peleforo Gon Coulibaly de Korhogo, UFR Sciences Biologique

\section{Moya Bi George, Doctorant}

Laboratoire de Botanique et Valorisation des Ressources Végétales, Sciences de la Nature, Université Nangui Abrogoua,Côte d'Ivoire

\section{Koné Mamidou Witabouna, Professeur}

Laboratoire de Botanique et Valorisation des Ressources Végétales, Sciences de la Nature, Université Nangui Abrogoua, Côte d'Ivoire Centre Suisse de Recherches Scientifiques en Côte d'Ivoire, Abidjan

\begin{abstract}
The hormonal deficiency due to age causes among others an increase of the fragility bone. To effectively control this disease, many authors have recommended the use of plants. In order to contribute to the reduction of this disease, this study was carried out with a view to promoting medicinal plants rich in phytoestrogens as an alternative solution against osteoporosis. For this purpose, the flavonoid and isoflavone contents of five plants from the Fabaceae family were determined by spectrophometric methods. Also the antifree radical potency power of these plants was evaluated using DPPH and ABTS methods. The anti-free radical potency of these plants was also evaluated using the DPPH and ABTS methods. This study indicates the presence of isoflavones and flavonoids in the plant extracts except for
\end{abstract}


Piliostigma thonningii roots. Uraria picta leaves have the highest flavonoid contents with $334.48 \pm 0.49 \mathrm{mg}$ EQ /100g dry matter, followed by Pericopsis laxiflora leaves with $210.27 \pm 0.71 \mathrm{mg} E Q / 100 \mathrm{~g}$ dry matter. These two plants have also high isoflavone contents with 66.61 $1.23 \mathrm{mg}$ GNT /100g DM and $21.05 \pm 1.15 \mathrm{mg}$ GNT /100g DM, respectively. The leaves, roots and stem bark of Pericopsis laxiflora showed the highest antioxidant activities with $95.86 \pm 9.27 \%, 91.02 \pm 0.58 \%$ and $99.99 \pm 0.0 \%$ of inhibition, respectively for the DPPH radical and $100 \pm 0,00 \%, 92,53 \pm 1,26 \%$ and $99,99 \pm 0,01 \%$ of inhibition, respectively for the ABTS radical. The results showed that these plants may have an interest in the prevention and/or treatment of osteoporosis.

Keywords: Medicinal Plants, Flavonoids, Isoflavones, Antioxidants, Fabaceae, Osteoporosis

\section{Introduction}

De nombreuses études prévoient que d'ici 2050 la population des personnes âgées de plus de 60 ans passera à deux milliards d'individus (Domenach H. 2008 ; OMS, 2018). Du fait du vieillissement de la population, le nombre de personnes âgées en perte d'autonomie augmentera sensiblement, notamment en raison d'une mobilité réduite. La prise en charge des maladies liées à l'âge ainsi que leur impact social et économique devront être reconsidérés par tous les pays, notamment les pays qui se veulent émergents. Dans son rapport de 2012, l'OMS demande aux professionnels de la santé de prendre des mesures appropriées pour prévenir les maladies non transmissibles, en promouvant, notamment, la santé et les comportements sains à tout âge pour éviter ou retarder l'apparition des maladies chroniques. En effet, les maladies qui touchent les personnes âgées sont entre autres les cardiopathies, les accidents vasculaires cérébraux, le cancer, le diabète, les maladies pulmonaires chroniques, la maladie d'Alzheimer, les affectations du système locomoteur telles que la sarcopénie, et l'ostéoporose (OMS, 2018). L'ostéoporose est la maladie la plus fréquente du squelette, caractérisée par une faible masse osseuse et une altération de la microarchitecture du tissu osseux. Elle entraîne une vulnérabilité aux fractures et une détérioration de la qualité de vie avec pour corolaire l'invalidité. L'ostéoporose touche généralement la femme ménopausée. L'accroissement continu de l'espérance de vie, l'urbanisation, l'occidentalisation du mode de vie et les coûts importants qu'elle pourrait engendrer font émerger cette affection comme un réel problème de santé publique. De nombreux médicaments de l'ostéoporose post-ménopausique existent, mais ils présentent néanmoins des limites (Sigrid, 2013). Le développement de nouveaux traitements s'avère donc essentiel. Le vieillissement et la physiopathologie de très nombreux organes dont l'os ont un lien avec le statut redox de l'organisme. Les espèces réactives de l'oxygène 
sont largement impliquées dans les complications associées au vieillissement (Krause, 2007) dont l'ostéoporose (Banfi et al., 2008, Salha, 2016). En effet, une augmentation du stress oxydant est associée à la perte osseuse ; ainsi l'augmentation de la production d'espèces réactives de l'oxygène est connue à la fois pour activer la différenciation des ostéoclastes et inhiber celle des ostéoblastes (Wauquier et al., 2009). Le statut redox de l'organisme est donc également un paramètre important pour l'acquisition d'un bon capital osseux. Les effets bénéfiques des phytœstrogènes tels les flavonoïdes (quercetine) et isoflavones dans la prévention et le traitement de l'ostéoporose sont prouvées (Douart, 1994 ; Banfi et al., 2008 ; Marie et Halbout, 2008 ; Al Hamdany et al., 2019). Ainsi, plusieurs travaux ont montré l'incidence et l'évolution de l'ostéoporose avec la consommation d'aliments riches en polyphénols (Chisari et al., 2019).

La recherche de solutions naturelles est au centre des préoccupations scientifiques puisqu'elles pourraient retarder la progression de la maladie et impacter significativement la qualité de vie de la population (Samo, 2012 ; Ahoua, 2017).

C'est dans ce contexte que s'inscrit le présent travail, centré sur la recherche de plantes médicinales comme une solution alternative contre l'ostéoporose. Plus spécifiquement il s'agit d'évaluer la teneur en flavonoïdes, en isoflavones et la capacité à réduire les espèces chimiques réactives de l'oxygène des plantes utilisées traditionnellement pour traiter des maladies qui seraient apparentées à l'ostéoporose telles que le rhumatisme et les fractures.

\section{Materiel Et Methodes}

\section{Sélection des espèces végétales étudiées}

Les échantillons sont des plantes de la famille des Fabaceae utilisées par les tradipraticiens pour le traitement des maladies qui pourraient avoir un lien avec l'ostéoporose. Cette famille a été sélectionnée à la suite d'enquêtes ethnobotaniques effectuées auprès de 28 guérisseurs de Brobo et Tié N'diékro, deux localités du centre de la Côte d'Ivoire. Lors de cette enquête plusieurs familles botanique ont été cités, ce sont les familles des Passifloraceae, des Zingiberaceae, des Sapindaceae, des Phyllanthaceae, des Connaraceae, des Chrysobalanaceae, des Sapindaceae, des Apocynaceae, des Pedaliaceae, des Canabaceae, des Lamiaceae, des Moraceae avec une espèce chacune, des Malvaceae, des Olacaceae avec 2 especes chacune et, des Fabaceae avec 5 espèces. Les espèces de la famille des Fabaceae qui ont été beaucoup citées par les praticiens de la médicine traditionnelle sont: Dalbergia hostilis Benth, Pericopsis laxiflora (Benth.) Meeuwen, Piliostigma thonningii (Schumach.) Milne-Redh, Pseudarthria hookeri Wight et Am. et Uraria picta (Jacq.) Desv. (Tableau I) 
Tableau I : Indications thérapeutiques des espèces végétales étudiées.

\begin{tabular}{ccc}
\hline Espèces végétales & Indications thérapeutiques & Organes utilisés \\
\hline Dalbergia hostilis (Benth) & Rhumatisme & $\begin{array}{c}\text { Feuille, } \\
\text { Ecorce de tige, }\end{array}$ \\
$\begin{array}{c}\text { Pericopsis laxiflora } \text { (Benth) } \\
\text { Piliostigma thonningii } \\
\text { (Schumach.) }\end{array}$ & Fracture, douleur articulaire et rhumatisme & Feuille et racines \\
Pseudarthria hookeri (Wight et & Fracture et migraine & Racine \\
Am.) & Fracture et douleur articulaire & Racine, \\
Uraria picta (Jacq.) Desv. & Fracture et rhumatisme & Feuille \\
\hline
\end{tabular}

\section{Préparation des extraits bruts}

Les organes ont été récoltés dans le mois de Mai à 8 heures 30 minutes puis pré-séchés, à l'ombre, sur le terrain avant leur acheminement à Abidjan. Une fois au laboratoire, les organes végétaux récoltés ont été séchés sous climatisation à $18^{\circ} \mathrm{C}$ pendant trois semaines puis pulvériser pour obtenir des poudres.

Pour la préparation des extraits bruts, $5 \mathrm{~g}$ de poudre ont été macérés dans $50 \mathrm{ml}$ de méthanol sous agitation mécanique pour permettre une meilleure extraction des composés chimiques. Après $24 \mathrm{~h}$ d'agitation, Le mélange est filtré et le filtrat obtenu a permis de réaliser les différents tests de détection, de dosage et d'évaluation de l'activité antioxydante. Un total de sept extraits, sur la base des organes utilisés, a été préparé.

\section{Test de détection des flavonoïdes et isoflavonoïdes (Réaction à la cyanidine)}

La détection des flavonoïdes et des isoflavones dans les extraits a été faite à l'aide de la réaction à la cyanidine. A $2 \mathrm{ml}$ de chaque extrait ont été ajoutés deux à trois copeaux de magnésium et $1 \mathrm{ml} \mathrm{d} \mathrm{HCl}$. La présence de flavonoïdes est caractérisée par différentes colorations consignées dans le tableau II (Ordonez et al., 2006).

Tableau II : composés mis en évidence par le test à la cyanidine

\begin{tabular}{ccccccc}
\hline Coloration & Rouge & $\begin{array}{c}\text { Jaune- } \\
\text { rougeâtre }\end{array}$ & $\begin{array}{c}\text { Rouge, } \\
\text { rouge- } \\
\text { violacé }\end{array}$ & $\begin{array}{c}\text { Rouge- } \\
\text { foncé, } \\
\text { violet, bleu }\end{array}$ & Jaune & Rose \\
\hline $\begin{array}{c}\text { Type de } \\
\text { flavonoïdes }\end{array}$ & Anthocyanes & Flavones & Flavonols & Flavonones & Isoflavones & Leucoanthocyanes \\
\hline
\end{tabular}

\section{Dosage des flavonoïdes et isoflavones}

La méthode du trichlorure d'aluminium $\left(\mathrm{AlCl}_{3}\right)$ a été utilisée pour quantifier les flavonoïdes dans les extraits, selon le protocole de Djeridane et al. (2006). L'absorbance est lue à $430 \mathrm{~nm}$ à l'aide du spectrophotomètre (HACH DR 2400) après 10 min d'incubation. La concentration des 
flavonö̈des dans l'extrait a été exprimée à partir de l'équation de la droite d'étalonnage : $\mathrm{Y}=0,0318 \mathrm{X}+0,0193$

Où Y est la valeur de l'absorbance et $\mathrm{X}$ la concentration en $\mu \mathrm{M}$ des flavonoïdes présents dans l'échantillon.

Selon Isabela et al. (2008), la quantification des isoflavones peut se faire aux longueurs d'ondes allant de 200 à $500 \mathrm{~nm}$. Dans ce travail, les mesures ont été faites à $400 \mathrm{~nm}$ en raison du spectrophotomètre disponible au laboratoire. La concentration des isoflavones dans les différents extraits a été exprimée à partir de l'équation de la droite d'étalonnage :

\section{(1) $Y=0,0143 X+0,0004$}

Où Y est la valeur de l'absorbance et $\mathrm{X}$ la concentration en $\mu \mathrm{M}$ des isoflavones présentes dans l'échantillon.

\section{Calcul des teneurs en flavonoïdes et isoflavones}

Les valeurs de la densité optique (DO) obtenus ont été exportées sous Excel pour être converties en $\mu \mathrm{g}$ d'équivalent de quercétine par mg d'extrait pour les flavonoïdes et en $\mu \mathrm{g}$ d'équivalent de génistéine par mg d'extrait pour les isoflavones. Les concentrations déterminées et le facteur de dilution ont permis de calculer la quantité des différents composés dans les extraits selon la formule suivante :

$$
\text { (2) } \mathrm{C}(\mathrm{mg} / 100 \mathrm{~g})=\left[\mathrm{Vs}(\mathrm{mL}) \times 10^{-3} / \mathrm{me}(\mathrm{g}) \times \mathrm{F} \times \mathrm{X}\right] \times 100
$$

Avec :

C : quantité du composé

Vs : volume de la solution prélevé pour l'extraction

me : masse de la poudre végétale

$\mathrm{F}:$ facteur de dilution

$\mathrm{X}$ : concentration de l'échantillon déterminée à partir de la droite d'étalonnage La quantité finale des flavonoïdes dans les différents extraits a été exprimée en mg d'équivalent de quercétine par $100 \mathrm{~g}$ d'extrait (mg EQ/100 g) et celle des isoflavones en $\mathrm{mg}$ d'équivalent de génistéine par $100 \mathrm{~g}$ d'extrait (mg GNT/100 g).

\section{Détermination des activités antioxydantes}

La détermination des activités antioxydantes a été faite au moyen de deux méthodes : au DPPH (1,1-diphenyl-2-picrylhydrazyl) et à l'ABTS (2,2azinobis (3- ethyl-benzothiazoline-6-sulfonic acid).

\section{Test au DPPH}

A $100 \mu \mathrm{L}$ d'extrait sont ajoutés $2500 \mu \mathrm{L}$ d'une solution méthanolique de $\mathrm{DPPH}$ à $0,4 \mathrm{Mm}$. Le mélange obtenu est ensuite incubé à l'obscurité pendant $30 \mathrm{~min}$ à $30{ }^{\circ} \mathrm{C}$ puis l'absorbance est mesurée à $517 \mathrm{~nm}$ contre un blanc (méthanol). La solution de DPPH est utilisée comme contrôle. Les essais 
ont été répétés trois fois. Le pourcentage d'inhibition du radical DPPH est calculé selon la formule suivante :

$$
\text { (3) \% Inhibition } \left.(\mathrm{DPPH})=\left[\left(\mathbf{A}_{0}-\mathbf{A}_{\mathbf{i}}\right) / \mathbf{A 0}\right)\right] \times 100
$$

$\mathrm{A}_{0}$ : absorbance du contrôle, $\mathrm{A}_{\mathrm{i}}$ : absorbance de l'extrait.

La capacité à réduire le radical DPPH est exprimée en mg d'équivalent de Trolox $\mathrm{g}^{-1} \mathrm{~d}^{\prime}$ extrait (mg TE g ${ }^{-1} \mathrm{~d}$ 'extrait) selon la formule de Wangcharoen et Morasuk, (2007) :

(4) Valeur $($ mgET.g-1 MS $)=[(($ A0-A1 $) /($ Pente $))($ V/v $)) /(\mathrm{m} \times 1000)]$

Avec A1 = Valeur de l'absorbance de l'échantillon, A0 = Valeur de l'absorbance du contrôle, Pente $=$ Pente de la droite de régression exprimant les valeurs des absorbances en fonction des différentes concentrations de Trolox, $\mathrm{V}=$ Volume total d'extrait préparé pour obtenir la solution mère, $\mathrm{v}=$ Volume de l'extrait prélevé pour le test, $\mathrm{m}=$ Masse de l'échantillon prélevé pour préparer la solution mère, 1000 = Facteur de conversion ( $\mu \mathrm{g}$ en mg).

\section{Test à l'ABTS}

Le cation $\mathrm{ABTS}^{+}$est obtenu par mélange (v/v) d'ABTS (7,0 Mm) et de persulfate de potassium $(2,6 \mathrm{mM})$, puis on laisse reposer à l'obscurité à température ambiante durant la nuit. Un volume de $1 \mathrm{~mL}$ de la solution $\mathrm{ABTS}^{+}$ est dilué avec $60 \mathrm{~mL}$ de méthanol pour obtenir une absorbance comprise entre 1 et 15 à $734 \mathrm{~nm}$. La solution est préparée juste avant les tests et ne peut être conservée. A $100 \mu \mathrm{L}$ de l'extrait méthanolique de plante sont ajoutés $2500 \mu \mathrm{L}$ de solution $\mathrm{ABTS}^{+}$puis le tout est incubé à l'obscurité pendant 2 heures.

Le pourcentage d'inhibition du radical $\mathrm{ABTS}^{+}$est calculé selon la formule suivante :

\section{(5) $\%$ Inhibition $\left.\left(\mathrm{ABTS}^{+}\right)=\left[\left(\mathbf{A}_{a^{-}} \mathbf{A}_{\mathrm{b}}\right) / \mathbf{A}_{\mathbf{a}}\right)\right] \times 100$}

$\mathrm{A}_{\mathrm{a}}$ : absorbance du contrôle, $\mathrm{A}_{\mathrm{b}}$ : absorbance de l'extrait.

La capacité à réduire le radical ABTS est exprimée en mg d'équivalent de Trolox $\mathrm{g}^{-1} \mathrm{~d}^{\prime}$ extrait (mg TE $\mathrm{g}^{-1} \mathrm{~d}^{\prime}$ extrait) selon la formule (4) décrite par Wangcharoen et Morasuk, (2007).

\section{Analyse statistique}

Les analyses ont été réalisées à l'aide du logiciel STATISTICA. Le test d'ANOVA a servi à comparer les moyennes des teneurs en flavonoïdes, isoflavones et les moyennes de pourcentages d'inhibition de l'ABTS et du DPPH des différents extraits. La plus petite différence significative a été fixée au seuil de 0,05 (Vessereau, 1992). 


\section{Resultats}

\section{Détection des flavonoïdes et isoflavones}

Sur les sept extraits d'organes végétaux étudiés, les flavonoïdes ont été détectés dans six extraits, soit dans quatre des plantes. Les extraits contenant les flavonoïdes ont été utilisés pour la recherche des isoflavones. Après investigation, les isoflavones ont été révélées dans cinq extraits issus des organes de plantes étudiés (Tableau III).

Tableau III : Composition qualitative des flavonoïdes et des isoflavones

\begin{tabular}{llll}
\hline Espèces végétales & Organes & Flavonoïdes & Isoflavones \\
\hline Dalbergia hostilis & Feuille & + & + \\
Pericopsis laxiflora & Feuille & + & + \\
& Ecorce de tige & + & + \\
Piliostigma thonningii & Racine & + & + \\
Pseudarthria hookeri & Racine & - & - \\
Uraria picta & Racine & + & - \\
\hline
\end{tabular}

(+) : présence ; (-) : absence

\section{Teneur en flavonoïdes et isoflavones}

Les teneurs en flavonoïdes et isoflavones sont présentées dans le tableau IV. Il existe une différence significative entre les différentes teneurs des composés recherchés $(\alpha=5 \%, P<0,001)$. Les valeurs des teneurs des plantes en flavonoïdes sont comprises entre 6,81et 334,48 mg EQ /100 g. L'extrait qui contient la plus forte teneur en flavonoïdes est celui obtenu avec

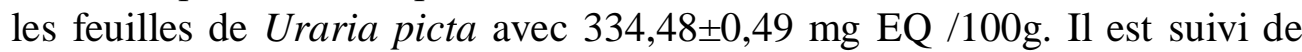

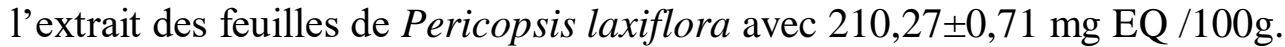
Quant aux isoflavones, les valeurs des teneurs sont comprises entre 0,32 et $66,61 \mathrm{mg}$ GNT /100g. L'extrait des feuilles de Uraria picta contient la plus forte quantité d'isoflavones et est suivi de celui des feuilles de Pericopsis laxiflora, avec respectivement $66,61 \pm 1,23 \mathrm{mg}$ GNT $/ 100 \mathrm{~g}$ et $21,05 \pm 1,15 \mathrm{mg}$ GNT /100g. Les isoflavones n'ont pas pu être déterminés dans l'extrait des racines de Pseudarthria hookeri. Les flavonoïdes et isoflavones n'ont pas été déterminés dans les racines de Piliostigma thonningii. 
Tableau IV: Teneurs en flavonoïdes et isoflavones (mg EQ/100 g d'extrait) des différents extraits de plantes étudiées

\begin{tabular}{|c|c|c|c|}
\hline \multirow[b]{2}{*}{ Espèce végétales } & \multirow[b]{2}{*}{ Organes étudiés } & \multicolumn{2}{|c|}{ Teneurs moyennes } \\
\hline & & Flavonoïdes (mg EQ /100g) & $\begin{array}{c}\text { Isoflavones } \\
\text { (mg GNT } \\
\text { /100g) }\end{array}$ \\
\hline \multirow{3}{*}{$\begin{array}{c}\text { Pericopsis } \\
\text { laxiflora }\end{array}$} & Racine & $6,81 \pm 0,19^{\mathrm{a}}$ & $0,32 \pm 0,09^{\mathrm{a}}$ \\
\hline & Feuille & $210,27 \pm 0,71^{\mathrm{e}}$ & $21,05 \pm 1,15^{\mathrm{b}}$ \\
\hline & Ecorce de tige & $38,42 \pm 0,45^{\mathrm{d}}$ & $0,33 \pm 0,09^{\mathrm{a}}$ \\
\hline \multirow{3}{*}{$\begin{array}{c}\text { Dalbergia hostilis } \\
\text { Uraria picta } \\
\text { Pseudarthria } \\
\text { hookeri }\end{array}$} & Feuille & $21,62 \pm 0,45^{\mathrm{b}}$ & $0,54 \pm 0,14^{\mathrm{a}}$ \\
\hline & Feuille & $334,48 \pm 0,49^{f}$ & $66,61 \pm 1,23^{b}$ \\
\hline & Racine & $24,64 \pm 0,44^{\mathrm{c}}$ & - \\
\hline \multicolumn{4}{|c|}{ Paramètres statistiques d'Anova } \\
\hline Dl & & 5 & 4 \\
\hline$F$ & & 237792,565 & 4317,92 \\
\hline$P$ & & $<0,001$ & \\
\hline
\end{tabular}

Les moyennes suivies des mêmes lettres sur la même colonne ne sont pas significativement différentes ; $d l$ : degré de liberté ; $F$ : valeur du test statistique ANOVA

\section{Pouvoir des extraits à piéger le radical DPPH ${ }^{\bullet}$}

Les extraits méthanoliques testés ont montré une importante activité antioxydante. Les résultats du potentiel des plantes à piéger le radical DPPH ${ }^{\bullet}$ sont consignés dans le Tableau V. Les activités de ces extraits sont comprises entre $9,17 \pm 0,58 \%$ et $99,99 \pm 0,0 \%$ d'inhibition. Les résultats montrent une différence significative (au seuil $\alpha=5 \%, \mathrm{P}<0,001$ ) entre les moyennes des valeurs des activités anti-radicalaires des extraits.

Les extraits les plus actifs sont ceux des feuilles, des racines et de l'écorce de tige de Pericopsis laxiflora avec respectivement 95,86 $\pm 9,27 \%$, $91.02 \pm 0,58 \%$ et $99,99 \pm 0,0 \%$ d'inhibition (Tableau V). Les feuilles de Pericopsis laxiflora présente la forte capacité antioxydante.

\section{Potentiel des extraits à inhiber le radical $\mathrm{ABTS}^{+}$}

Les résultats des activités des extraits sur le radical $\mathrm{ABTS}^{+}$sont présentés dans le tableau V. Les extraits des feuilles, des écorces de tige et des racines de Pericopsis laxiflora sont les plus actifs. Ils inhibent le radical $\mathbf{A B T S}^{+}$respectivement à $100 \pm 0,00 \%, 99,99 \pm 0,01$ et $92,53 \pm 1,26 \%$. Les feuilles et des écorces de tige de $P$. laxiflora présentent les plus fortes capacités antioxydantes avec respectivement $371,51 \pm 0,01$ et $371,53 \pm 0,01 \mathrm{mgET} \cdot \mathrm{g}^{-1} \mathrm{MS}$ $\pm \mathrm{SD}$ 
Tableau V : Activités antioxydantes des extraits des plantes étudiées

\begin{tabular}{|c|c|c|c|c|c|}
\hline \multicolumn{6}{|c|}{$\begin{array}{c}\text { Potentiel } \\
\text { Antioxydant }\end{array}$} \\
\hline Espèces végétales & & $\begin{array}{c}\text { \% d'inhibition } \\
\text { ABTS }\end{array}$ & $\begin{array}{c}\text { \% d'inhibition } \\
\text { DPPH }\end{array}$ & $\begin{array}{c}\text { Capacité } \\
\text { antioxydante } \\
\text { ABTS } \\
\text { (mgET.g } \\
\text { MS } \pm \text { SD) }\end{array}$ & $\begin{array}{c}\text { Capacité } \\
\text { antioxydante } \\
\text { DPPH }(\mathbf{m g E T} . \\
\text { MS } \pm \text { SD })\end{array}$ \\
\hline $\begin{array}{c}\text { Dalbergia } \\
\text { hostilis(Benth) }\end{array}$ & Feuille & $39,87 \pm 2,51^{b}$ & $18,19 \pm 1,46^{\mathrm{b}}$ & $140,79 \pm 1,35^{\mathrm{b}}$ & $57,14 \pm 4,76^{\mathrm{b}}$ \\
\hline $\begin{array}{c}\text { Pericopsis } \\
\text { laxiflora (Benth) }\end{array}$ & $\begin{array}{l}\text { Feuille } \\
\text { Ecorce } \\
\text { de tige }\end{array}$ & $\begin{array}{l}100 \pm 0,00^{\mathrm{d}} \\
99,99 \pm 0,01^{\mathrm{d}}\end{array}$ & $\begin{array}{l}95.86 \pm 9,27^{\mathrm{e}} \\
99,99 \pm 0,01^{\mathrm{e}}\end{array}$ & $\begin{array}{l}371,51 \pm 0,01^{\mathrm{e}} \\
371,53 \pm 0,01^{\mathrm{e}}\end{array}$ & $\begin{array}{l}341,45 \pm 9,27^{\mathrm{e}} \\
301,57 \pm 0,01^{\mathrm{d}}\end{array}$ \\
\hline & Racine & $92.53 \pm 1,26^{\mathrm{d}}$ & $91.02 \pm 0,58^{\mathrm{c}}$ & $341,79 \pm 4,66^{\mathrm{d}}$ & $291,59 \pm 1,86^{\mathrm{cd}}$ \\
\hline $\begin{array}{c}\text { Piliostigma } \\
\text { thonningii }\end{array}$ & Racine & $16,31 \pm 1,35^{\mathrm{a}}$ & $9,17 \pm 0,58^{\mathrm{a}}$ & & $31,16 \pm 0,30^{\mathrm{a}}$ \\
\hline $\begin{array}{c}\text { (Schumach.) } \\
\text { Pseudarthria } \\
\text { hookeri (Wight et }\end{array}$ & Racine & $37,50 \pm 0,13^{b}$ & $10,19 \pm 1,80^{\mathrm{a}}$ & $53,34 \pm 1,57^{\mathrm{a}}$ & $33,38 \pm 5,88^{a}$ \\
\hline $\begin{array}{c}\text { Am.) } \\
\text { Uraria picta } \\
\text { (Jacq.) DC. }\end{array}$ & Feuille & $75,19 \pm 7,89^{c}$ & $88,66 \pm 0,57^{\mathrm{c}}$ & $\begin{array}{l}138,49 \pm 0,49^{\mathrm{b}} \\
294,40 \pm 3,05^{\mathrm{c}}\end{array}$ & $284,02 \pm 1,82^{\mathrm{c}}$ \\
\hline $\begin{array}{c}\text { Paramètre } \\
\text { statistique d'Anova }\end{array}$ & $\mathrm{dl}$ & 6 & 6 & 6 & 6 \\
\hline & $\begin{array}{l}F \\
P\end{array}$ & $\begin{array}{l}343,557 \\
<0,001\end{array}$ & $\begin{array}{l}3075,12 \\
<0,001\end{array}$ & $\begin{array}{l}10120,44 \\
<0,001\end{array}$ & $\begin{array}{l}2845,143 \\
<0,001\end{array}$ \\
\hline
\end{tabular}

Les moyennes suivies des mêmes lettres sur la même colonne ne sont pas significativement différentes ; $d l$ : degré de liberté ; $F$ : valeur du test statistique ANOVA

\section{Discussion}

Cette étude a été menée afin d'identifier les potentialités phytœstrogènes de cinq plantes, issues de la famille des Fabaceae. En effet, selon Wink (2013) les Fabaceae sont riches en polyphénols (flavonoïdes et isoflavones) et elles renfermeraient des plantes ayant des propriétés bénéfiques pouvant améliorer l'état de santé des personnes souffrant de l'ostéoporose et des maladies apparentées. Or la carence en ces éléments entraîne des modifications du métabolisme osseux, à l'origine des processus de décalcification (Riggs et al., 2002; Anses, 2012). Les polyphénols pourraient exercer des effets protecteurs contre les maladies hormonodépendantes telle que l'ostéoporose en modulant la réponse aux œstrogènes endogènes (Scalbert et Williamson, 2000). Les flavonoïdes ont la capacité de piéger les radicaux libres (Mpondo et al., 2012 ;Bakchiche and Gherib 2014) générés par les organismes suite aux agressions (cigarettes, polluants, infections...) favorisant le vieillissement cellulaires (Burda et Oleszek, 2001). Quant aux isoflavones, ils ont la capacité de se lier aux récepteurs œstrogéniques pour manifester une activité phytœstrogénique (Barnes et al., 
2000; Hwang et al., 2006). Aussi, les cellules impliquées dans le remodelage osseux étant étroitement régulées par de nombreux facteurs hormonaux, les phytœstrogènes pourraient avoir un effet contre l'ostéoporose (Dang et Lowik, 2005 ; Marie et Halbout, 2008). Les phyto-œstrogènes (isoflavones) agissent sur l'activité du tissu osseux en inhibant l'action destructrice de l'os par les cellules ostéoclastes et en stimulant l'activité des ostéoblastes pour la synthèse d'une matrice osseuse abondante. Les phyto-œstrogènes agissent aussi sur l'absorption intestinale du calcium (Omi et al., 1994; Arjmandi et al., 2002) et sur la diminution de son excrétion urinaire (Kopcewicz, 1971), entrainant l'augmentation du taux circulant de calcium dans le sang. Par leurs propriétés phytœstrogéniques, les isoflavones ont suscité beaucoup d'intérêt pour le soulagement de l'ostéoporose et des troubles liés à la ménopause, tels que les bouffées de chaleur (Penotti et al., 2003 ; Howes et al., 2006). La présence de ces éléments dans les Fabaceae pourrait être à l'origine de leur efficacité et donc de leur sollicitation dans le traitement des maladies liées aux os. L'activité antiostéoporose d'autres plantes de cette famille dont Arachis hypogaea, Phaseolus vulgaris et Millettia macrophylla a déjà été rapportée (Kouakou et al., 2008 ; Zingue et al.,2014).

Les flavonoïdes sont retrouvés dans les feuilles de Dalbergia hostilis, les feuilles, écorce de tige et racines de Pericopsis laxiflora, les racines de Pseudarthria hookeri et les feuilles de Uraria picta.

Quant aux isoflavones, à l'exception des racines de Piliostigma thonningii et Pseudarthria hookeri, ils sont retrouvés dans les organes sélectionnés des autres plantes étudiées.

On note des quantités importantes de flavonoïdes et d'isoflavones dans les feuilles de Uraria picta avec respectivement $334,48 \pm 0,49 \mathrm{mg}$ EQ $/ 100 \mathrm{~g}$ et $66,61 \pm 1,23 \mathrm{mg}$ GNT $/ 100 \mathrm{~g}$ et de Pericopsis laxiflora, avec respectivement $210,27 \pm 0,70 \mathrm{mg}$ EQ / 100g et 21,05 $\pm 1,15$. mg GNT / 100g. Ces résultats corroborent ceux de plusieurs auteurs. En effet, Koevi et al., 2015 et Koffi et al., 2015 ont indiqué la présence de flavonoïdes dans les feuilles de Pericopsis laxiflora. Aussi, Hari et al., (2014) ont montré la présence de flavonoïdes dans l'extrait méthanolique des feuilles, tige et racine de Uraria picta. En 2013, Odubanjo et al. ont indiqué également la présence de flavonoïdes dans les feuilles de cette même plante. Par ailleurs, deux isoflavones le 5,7-dihydroxy2'-methoxy-3',4'-methylenedioxyisoflavanone et 4',5'-dihydroxy-2';3'dimethoxy-7-(5-hydroxyoxychromen-7yl)-isoflavanone ont déjà été isolés des racines de Uraria picta (Rahman et al., 2007). La présence de flavonoïdes constatés dans les feuilles de Pseudarthria hookeri dans cette étude confirme les résultats de Dzoyem et al. en 2018 qui ont identifié et isolé des flavonoïdes de la plante entière. La présence des flavonoïdes et isoflavones et leurs taux élevés pourraient conférer à ces deux plantes des effets sur le métabolisme 
osseux en faveur de la calcification. Elles devraient donc être explorées davantage dans la recherche de solutions alternatives contre l'ostéoporose.

Dans ce travail, la capacité des plantes à piéger les radicaux DPPH et ABTS a été évaluée. Ainsi pour le DPPH, les feuilles, écorces de tige et racines de Pericopsis laxiflora ont montré les plus forts pourcentages d'inhibition avec respectivement $95,86 \% ; 99,99 \%$ et $91.02 \%$ d'inhibition. Pour l'ABTS, les feuilles et les racines, écorces de tiges et racines de Pericopsis laxiflora possèdent les plus fortes activités antioxydantes avec respectivement à $100 \pm 0,00 \%, 99,99 \pm 0,01$ et 92,53 $\pm 1,26 \%$. Il ressort que les écorces de tige de Pericopsis laxiflora présentent le plus fort pouvoir antioxydant. Koffi et al. (2015) ont indiqué la présence de polyphénols dans les feuilles de Pericopsis laxiflora. La capacité réductrice de ces plantes pourrait donc être due principalement aux composés phénoliques présents dans ces dernières (Odabasoglu et al., 2004). L'activité antioxydante des feuilles de Pericopsis laxiflora obtenue dans ce travail confirme les résultats de Koevi et al. (2015) qui ont montré l'activité antioxydante de l'extrait éthanolique des feuilles de cette même plante. En outre, Patel et al. (2011) ont montré l'activité antioxydante de l'extrait de Uraria picta contre les radicaux ABTS et DPPH. Odubanjo et al. (2013) ont également montré l'activité antioxydante contre le radical ABTS de l'extrait aqueux de cette plante. Les résultats de ce travail viennent donc confirmer l'activité antioxydante de cette plante.

Le statut redox de l'organisme est un paramètre fondamental pour le capital osseux. En effet, une augmentation du stress oxydant est associée à de nombreuses situations de perte osseuse et l'augmentation de la production d'espèces réactives à l'oxygène est connue à la fois pour activer la différenciation des ostéoclastes et inhiber celle des ostéoblastes (Wauquier et al., 2009). Uraria picta et Pericopsis laxiflora par leurs activités antioxydantes pourraient jouer un rôle dans l'inhibition des ostéoclastes et l'activation des ostéoblastes. Ces plantes pourraient donc avoir un rôle protecteur contre l'ostéoporose. Elles devraient être explorées davantage dans la recherche de solution contre l'ostéoporose.

\section{Conclusion}

Ce travail a tenté d'apporter des réponses quant à la mise au point d'alternatives pour la prise en charge de l'ostéoporose qui affecte le plus souvent les personnes âgées. La présente étude indique la présence d'isoflavones et de flavonoïdes dans les extraits de Pericopsis laxiflora, Dalbergia hostilis, Uraria picta et Pseudarthria hookeri. Tous ces extraits possèdent une activité antioxydante. Cependant Uraria picta et Pericopsis laxiflora méritent d'être explorés davantage pour leurs activités remarquables. Ils pourraient donc jouer un rôle dans la prévention et/ou le traitement de l'ostéoporose. 


\section{Remerciements}

Nous remercions sincèrement le Centre Suisse de Recherches Scientifiques en Côte d'Ivoire (CSRS) pour l'assistance technique. Aussi nous sommes reconnaissants à l'Initiative DELTAS Africa [Afrique One-ASPIRE /DEL-15-008], [107753/A/15/Z] qui soutient Dr Ahoua Constant pour ses études postdoctorales.

\section{References:}

1. Ahoua A. R. C. (2017). Plantes consommées par les chimpanzés (Pan troglodytes verus, Blumenbach 1779) du Parc National de Taï, Côte d'Ivoire : activités biologiques, potentiel nutritif et investigation phytochimique de Beilschmiedia mannii (Meisn.) Robyns \& R. Wilczek (Lauraceae). Thèse de Doctorat, Université Nangui Abrogoua (Côte d'Ivoire): $255 \mathrm{p}$.

2. Al Hamdany A. K., Al-Khatib A. R. \& Al-Sadi H. I., (2019) an overview of the benefical effects of quercitin on bone. International Medical Journral, 2 (26) : 142-145.

3. Anses (2012). "Composition nutritionnelle des aliments. TABLE Ciqual 2012." http://www.anses.fr/TableCIQUAL/index.htm. consulté le 02 octobre 2017.

4. Arjmandi B., Khalil D. \& Hollis B. (2002). Soy protein: its effects on intestinal calcium transport, serum vitamin $\mathrm{D}$, and insulin-like growth factor-I in ovariectomized rats. Calcified tissue international, 70 (6): 483-487.

5. Bakchiche B. \& Gherib A. (2014). Activités antioxydantes des polyphenols extraits de plantes médicinales de la pharmacopée traditionnelle d'Algérie. International Journal of Innovation and Applied Studies. 9167-9172.

6. Banfi G., Iorio E. L. \& Corsi M. M. (2008). Oxidative stress, free radicals and bone remodeling. Clinical chemistry and laboratory medicine, 46 (11): 1550-1555.

7. Barnes S., Boersma B., Patel R., Kirk M., Darley-Usmar V., Kim H. \& Xu J. (2000). Isoflavonoids and chronic disease: mechanisms of action. Biofactors, 12 (1-4): 209-215.

8. Burda S. \& Oleszek W. (2001). Antioxidant and antiradical activities of flavonoids. Journal of agricultural and food chemistry, 49 (6): 27742779.

9. Chisari E., Shivappa N. \& Vyas S. (2019). Polyphenol- rich foods and osteoporosis. Current Pharmaceutical Design, 25 (22): 2459-2466.

10. Dang Z. C. \& Lowik C. (2005). Dose-dependent effects of phytoestrogens on bone. Trends in Endocrinology \& Metabolism, 16 (5): 207-213. 
11. Djeridane A., Yousfi M., Nadjemi B., Boutassouna D., Stocker P. \& Vidal N. (2006). Antioxidant activity of some Algerian medicinal plants extracts containing phenolic compounds. Food chemistry, 97 (4): 654-660.

12. Domenach H. 2008. « Les grandes tendances démographiques et l'environnement : l'enjeu d'une planète viable », Mondes en développement, 2 (142), p. 97-111. DOI : 10.3917/med.142.0097. URL : https://www.cairn.info/revue-mondes-en-developpement2008-2-page-97.htm

13. Douart J.-P. (1994). L'oligothérapie en pathologie fonctionnelle Données scientifiques et cliniques., Edition Maloine, 293p.

14. Dzoyem J. P., Tchamgoue J., Tchouankeu J.C., Kouan S.F., Choudhary M. I. \& Bakowsky U. (2018). Antibacterial activity and cytotoxicity of flavonoids compounds isolated from Pseudarthria hookeri Wight et Arn. (Fabaceae). South African Journal of Botanique, 114: 100-103.

15. Hari O. S., Anjana S., Naseer M. \& Santos K. C. (2014). Phytochemical screening and elemental analysis in different plant parts of Uraria picta Desv. : A Dashmul species. Journal of chemical and pharmaceutical Research, 6 (5):756-760.

16. Howes L. G., Howes J. B. \& Knight D. C. (2006). Isoflavone therapy for menopausal flushes: a systematic review and meta-analysis. Maturitas, 55 (3): 203-211.

17. Hwang C. S., Kwak H. S., Lim H. J., Lee S. H., Kang Y. S., Choe T. B., Hur H. G. \& Han K. O. (2006). Isoflavone metabolites and their in vitro dual functions: they can act as an estrogenic agonist or antagonist depending on the estrogen concentration. The Journal of steroid biochemistry and molecular biology, 101 (4): 246-253.

18. Isabela D.C.C., Fernão C.B., Cristina D.V-S., Elzíria A.N., Gérson A. \& Pianetti E.L.M.M-C. (2008). Quantitation of genistein and genistin in soy dry extracts by UV-Visible. Química Nova, 31 (8): 1933-1936.

19. Kopcewicz J. (1971). Estrogens in developing bean (Phaseolus vulgaris) plants. Phytochemistry, 10 (7): 1423-1427.

20. Kouakou K., Kati-Coulibaly S. \& Mangue N'T. E. J. (2008). Effets bénéfiques des extraits d'Arachis hypogaea (Fabaceae) et de Phaseolus vulgaris (Fabaceae) sur la perte osseuse chez un modèle animal d'ostéoporose. Phytothérapie, 6 (1): 5-12.

21. Koffi A. J., Bla K. B., Yapi H. F., Bidie A. P. \& Djaman A. J. (2015). Phytochemical screening of some plants in Côte d'Ivoire and evaluation of their extraction efficiency. International Journal of Pharmacognosy and Phytochemical Research, 7 (3): 563-569. 
22. Koevi K.-K. A., Millogo V., Hzounda Fokou J. B., Sarr A., Ouedraogo G.A. \& Bassene E. (2015). Phytochemical analysis and antioxidant activities of Combretum molle and Pericopsis laxiflora. Int. J. Biol. Chem. Sci., 9 (5): 2423-2431.

23. Krause K.-H. (2007). Aging: a revisited theory based on free radicals generated by NOX family NADPH oxidases. Experimental gerontology, 42 (4): 256-262.

24. Marie P. \& Halbout P. (2008). OPG/RANKL-Implication et cible thérapeutique dans l'ostéoporose Médecine / Sciences, . 24 (1): 105110.

25. Mpondo E., Dibong S. D., Ladoh Y. C. F., Priso R. J. \& Ngoye A. (2012). Les plantes à phénols utilisées par les populations de la ville de Douala. Journal of Animal \&Plant Sciences, 15: 2083-2098.

26. Odabasoglu, F., Aslan, A., Cakir A., Suleyman H., Karagoz Y., Halici M. \& Bayir Y. (2004). Comparison of ant ioxidant activity and phenolic content of three lichen species. Phytotherapy Research, 18(11): 938-941.

27. Odubanjo V. O., Oboh G \& Ibukun E. O. (2013). Antioxidant and anticholinesterase activities of aqueous extract of Uraria picta (Jacq.) DC. African Journal of Pharmacy and Pharmacology, 7(41), 27682773.

28. Omi N., Aoi S., Murata K. \& Ezawa I. (1994). Evaluation of the effect of soybean milk and soybean milk peptide on bone metabolism in the rat model with ovariectomized osteoporosis. Journal of nutritional science and vitaminology, 40 (2): 201-211.

29. OMS (2018). Vieillissement et santé. www.who.int/fr/newsroom/fact-sheets/detail/ageing-and-health. Consulté le 08/10/2019.

30. Ordonez A., Gomez J. \& Vattuone M. (2006). Antioxidant activities of Sechium edule (Jacq.) Swartz extracts. Food chemistry, 97 (3): 452458.

31. Patel B. D., Kamariya Y. H. \& Patel M. B. (2011). Free radical scavenging potential OF ethanolic extract of Uraria picta linn. Pharmacologyonline, 2: 134-145

32. Penotti M., Fabio E., Modena A. B., Rinaldi M., Omodei U. \& Viganó P. (2003). Effect of soy-derived isoflavones on hot flushes, endometrial thickness, and the pulsatility index of the uterine and cerebral arteries. Fertility and Sterility, 79 (5): 1112-1117.

33. Rahman M. M., Gibbons S. \& Gray A. I. (2007). Isoflavones from Uraria picta and their antimicrobial activity. Phytochemistry, 68:16921697. 
34. Riggs B. L., Khosla S. \& Melton III L. J. (2002). Sex steroids and the construction and conservation of the adult skeleton. Endocrine reviews, 23 (3): 279-302.

35. Salha B. (2016). Comportement in vitro et invivo de verres composites poreux : assimilation osseuse, explorations physiologiques et physicochimique. Thèse de Doctorat, Université de Rennes 1 et de l'Université de Sfax: 310 p.

36. Samo R. (2012). Diet and Aging. Oxidative Medicine and Cellular Longevity, 2012 : 20p.

37. Sigrid A. (2013). Rationnel de développement et strategie d'enregistrement d'Odanacatib dans le traitement de l'ostéoporose postménopausique. Thèse de Doctorat en pharmacie, Université Joseph Fourier, faculté de pharmacie de grenoble: 119 p.

38. Marie P. \& Halbout P. (2008). OPG/RANKL-Implication et cible thérapeutique dans l'ostéoporose Médecine / Sciences, . 24 (1): 105110.

39. Vessereau A. 1992. Méthodes Statistiques en Biologie et en Agronomie. Tec et doc Lavoisier: Paris, 337p.

40. Wangcharoen, W., \& Morasuk, W. (2007). Antioxidant capacity and phenolic content of some Thai culinary plants. Maejo International Journal of Science and Technology, 1(2), 100-106.

41. Wauquier F., Leotoing L., Coxam V., Guicheux J. \& Wittrant Y. (2009). Oxidative stress in bone remodelling and disease. Trends in molecular medicine, 15 (10): 468-477.

42. Wink M. (2013). Evolution of secondary metabolites in legumes (Fabaceae). South African Journal of Botany, 89: 164-175.

43. Zingue S., Njamen D., Mvondo M. A. \& Nde C. B. M. (2014). Preventive effects of the methanol soluble fraction of Millettia macrophylla Benth (Fabaceae) on an osteoporosis-like model of ovariectomized Wistar rats. Journal of Complementary and Integrative Medicine, 11 (2): 83-92. 